\title{
Expression of VEGF receptors VEFGR-1 and VEGFR-2, angiopoietin receptors Tie-1 and Tie-2 in chorionic villi tree during early pregnancy
}

\section{Ramazan Demir}

\author{
Department of Histology and Embryology, Faculty of Medicine, Akdeniz University, Antalya, Turkey.
}

\begin{abstract}
The aim of this study was to determine the expression of VEGF and its receptors in placentas from normal pregnancies between 22 days p.c. and 48 days p.c. of very early pregnancy. Placental tissues carried out from 19 pregnant women were examined. Immunohistochemical technique, electron microscopy were employed to evaluate the factors expression. In the new developing mesenchymal villi and immature intermediate villi VEGF and its receptors VEGFR-1 and VEGFR-2 immunoreactivity was detected in all the placental components, while in the stem villi and in the chorionic plate with large vessels only in some components. In the mesenchymal villi and immature intermediate villi VEGFR-1 and -2, and angiopoietin receptors Tie-1 and -2 immunoreactivity was dominantly observed in the heamangiogenic cells and cells cords, whereas the matured villi showed immunoreactivity only in other components. The ultrastructural findings were higher in respect to the all of the early pregnancy days. The placental samples from all of pregnancies, showed the VEGF and its receptors in optimal expression levels, whereas the angiopoietin receptors Tie-1 and -2 showed a higher expression levels in respect to other study factors. The receptors protein levels increased from the early days to the advanced days of gestation, but this alteration was not significant. The intensity of the immunolabeling for these proteins were not significant compared to to each other of gestatin days were examined. These findings demonstrated that a dysregulation of the placental expression of the VEGF and its receptors related to the different degrees of the gestational periods. Probably, this event may be related to complete vasculugenesis and angiogenesis in placental villi.
\end{abstract}

Key words: angiogenesis, vasculogenesis, early pregnancy, haemangiogenic cells, human placenta

\section{Introduction}

The terms angiogenesis and vasculogenesis have been used synonymously and both terms have been used to characterize the general formation and development of blood vessels. However, there is still no agreement on the exact meaning of these two terms, vasculogenesis and angiogenesis. Although they were well defined (Cogle and Scott, 2004), their characteristic meanings were not appropriately and uniformly utilized. Angiogenesis has been used to describe immigration (or migration) of angioblasts, development of endothelial cells from pre-existing blood vessels (Benirschke and Kaufmann 2000; Charnock-Jones et al., 2004), and sprouting of endothelial cells (Pardanaud et al., 1989).

Correspondence: R. Demir, Department of Histology and Embryology, Faculty of Medicine, Akdeniz University, Antalya, Turkey; tel./fax.: (+090) 2422274486,

e-mail: rdemir@kdeniz.edu.tr
In all types of angiogenesis, either under physiological or pathological conditions, endothelial cell activation is the first process to take place, leading to increased vascular permeability. The main goal of this process is to supply new blood. Various factors affect angiogenesis and vasculogenesis by autocrine or paracrine and as wall as directly or indirectly stimulating proliferation and differentiation of endothelial precursor cells. (Millauer et al., 1993; Hanahan and Folkman, 1996; Ferrara, 2004).

So far, four different VEGF subtypes have been described, VEGF-A to VEGF-D (Tammela et al., 2005). When it was first described VEGF has been thought to act as a vascular permeability factor (VPF) (Senger et al., 1983). The first identified VEGF-A is a disulphide-linked homodimeric glycoprotein that plays an important role in the vasculogenesis, angiogenesis, vascular permeability and vasodilatation (Cheung, 1997; Torry and Torry, 1997; Kingdom et al., 2000; Vuorela et al., 1997)..VEGF is a heparin-binding 
growth factor known to bind directly to its surface receptors. The receptors for the VEGF family are VEGFR-1/Flt-1, VEGFR-2/KDR/Flk-1 and VEGFR3/Flt-4 have been identified in placental tissues (Cooper et al., 1995; Shore et al., 1997; Leach et al., 2002; Vuckovic et al., 1996;). VEGFR-1 is the receptor for PIGF, VEGF-A, and VEGF-B (Terman et al., 1992); while VEGFR-2 for VEGF-A, VEGF-C and VEGF-D (Joukov et al., 1996). VEGFR-3 is the receptor for VEGF-C and VEGF-D which seems to play a role in regulation of angiogenesis and lymphangiogenesis $(\mathrm{Gu}$ et al., 2006).

Multiple studies have shown that both receptors are clearly involved in capillary formation and vascular development during embryogenesis (Shalaby et al., 1995; Ferrara, 2000; Hiratsuka et al., 2005).

VEGF and its two receptors, VEGFR-1 (Hanahan, 1997) and VEGFR-2 (Terman et al., 1992; Millauer et al., 1993) are expressed in the endothelium of blood vessels and contribute to vascular development and regulation of vascular permeability (Terman et al., 1992). The vasculogenic importance of two newly detected receptors tryosine kinases Tie-1 and Tie-2, angiopoietin receptors, has been studied (Sato et al., 1995; Suri et al., 1996; Maisonpierre et al., 1997; Kayisli et al., 2006).

Recently, placental vasculogenesis and angiogenesis were reviewed and discussed in detail with different aspects (Leach et al., 2002; Kaufmann et al., 2004; Mayhew et al., 2004).

Another closely related protein, placenta growth factor (PIGF), is abundantly expressed in the placenta ( Park et al., 1994) and has been extensively investigated within the last decade (Dvorak et al., 1995; Vuorela et al., 1997; Clark et al., 1998; Szukiewicz et al., 2005).

In VEGF knockout mice (Ferrara, 2000) and in the absence of VEGFR-2 no differentiation of precursor cells into endothelial cells occurs during vasculogenesis (Shalaby et al., 1995). On the other hand, a mutation in the locus of VEGFR-1 (Fong et al., 1995) does not prevent generation of endothelial cells but results in endothelial disorganization and abnormal vessel formation during early embryo development. Knockout experiments in mice have highlighted the importance of VEGFR-2 for recruitment and early differentiation of haemangiogenic cells into fetoplacental capillaries (Shalaby et al., 1995) and of VEGFR-1 for subsequent arrangement of early endothelial cells to form endothelial tubes (Fong et al., 1995; Fong et al., 1999).

Moreover, the early appearance of macrophages (Hofbauer cells) in the villous core which have been affected the villous vascularisation (Demir and Erbengi, 1984) and described to regulate distinct trophoblast functions (Cervar et al., 1999) and to express angiogenic growth factors (VEGF) (Demir et al., 2004), suggests a paracrine role for these cells during the first stages of vasculogenesis (Demir et al., 1997; Demir et al., 2006, 2007; Seval et al., 2007).

The aim of the present study was to investigate the expression profile of VEGF and its receptors, VEGFR1 and-2 with angiopoietin receptors Tie-1 and Tie-2; and to evaluate the molecular and morphological steps during vasculogenesis and angiogenesis in the early human placenta.

\section{Materials and Methods}

Samples of human placental tissues were obtained after legal termination of pregnancy by curettage for medical or psychosocial reasons, which were unlikely to affect placental structure and function. None of the pregnant women received hormone treatment; all were normal pregnancies. Tissues were supplied from the Department of Obstetrics and Gynecology, Akdeniz University, and the Clinic of Obstetrics and Gynecology, Government Hospital, Antalya. Written consent was obtained from patients before the procedure. Consent forms and protocols to use the tissue were approved by the Ethical Committee of Medical Faculty of Akdeniz University.

Tissue collection and storage. Samples of human placental tissues from 29 women were studied. Tissue samples were obtained from curettage material of uncomplicated normal pregnancies during very early stages of pregnancy (22 $2^{\text {nd }} 48^{\text {th }}$ day post conception, $p$. c.). Samples were classified as described in our previous study (Demir et al., 1989) using embryonic developmental details following the Carnegie classification (O'Rahilly and Tucker, 1973). Immediately after vacuum curettage (no application of prostaglandins) placental tissues were collected and divided into three pieces in order to perform immunohistochemistry, western blot and transmission electron microscopy. A total of four groups [each defining different gestational ages; days 22-28 (4th week, $\mathrm{n}=11$ ), days 29-35 (5th week, $\mathrm{n}=8$ ), days 36-42 (6th week, $\mathrm{n}=6$ ), days 43-49 (7th week, $n=4)$ ] of placental tissues were used for semi-quantitative evaluation of immunohistochemical staining. Additionally to these samples, three samples for $8^{\text {th }}$ week pregnancy were used only for western blotting analyses.

Electron microscopy. Samples of human placental tissues were fixed by immersion in $2.5 \%$ glutaraldehyde in $0.1 \mathrm{M}$ cacodylate buffer $(\mathrm{pH} 7.4)$ at room temperature for $4 \mathrm{~h}$ and post-fixed in $1 \%$ phosphate-buffered osmium tetroxide for $2 \mathrm{~h}$ (Demir, 1980). The specimens were dehydrated in ascending concentrations of ethanol and embedded in Araldite-epoxy resin. Semi-thin sections were stained with toluidine blue. Ultra-thin sections were double-stained with uranyl acetate and lead citrate (Demir et al., 2005) and examined with electron microscopes (Zeiss M10 and Leo 906).

Immunohistochemistry. Placental tissues were fixed in $4 \%$ paraformaldehyde for 4-6 h and embedded in paraffin. Serial sections were collected on poly-L-lysine coated slides (Sigma, St. Louis, MO, USA), dewaxed, dehydrated, and placed in citrate buffer ( $\mathrm{pH} \mathrm{6;1} 1$ distilled water; $2.1 \mathrm{~g}$ citric acid, $15 \mathrm{ml} \mathrm{NaOH}$ ). Immunohistochemical detection procedures have been described previously (Demir et al., 2002), and were slightly modified for our antibodies. To unmask antigens, an antigen retrieval procedure was performed by treating the samples in a microwave oven at $750 \mathrm{~W}$ for $5 \mathrm{~min}$ twice. After cooling for $20 \mathrm{~min}$ at room temperature, the sections were washed in phosphate buffered saline (PBS; pH 7.4). To remove endogenous peroxidase activity, sections were kept in $3 \%$ hydrogen peroxide (Dako A/S, Glostrup, Denmark) for $30 \mathrm{~min}$ 
and washed with PBS three times. The following primary antibodies were used: monoclonal mouse anti-human VEGF (1:100; clone C-1, Santa Cruz Biotechnology Inc, Santa Cruz Biotechnology, CA, USA), polyclonal rabbit anti-VEGFR-1 /Flt-1 (1:200, cat \# C17, Santa Cruz Biotechnology), monoclonal mouse antiVEGFR-2 (Flk-1/KDR) (1:200; clone A-3, Santa Cruz Biotechnology) (Demir et al., 2004). Rabbit anti Tie-1 (1:200, polyclonal, SC342; Santa Cruz Biotechnology), rabbit anti Tie-2 (1:200, polyclonal, SC-324; Santa Cruz Biotechnology), monoclonal mouse anti-human Tie-1 $(25 \mu \mathrm{g} / \mathrm{ml}$, MAB619, R\&D Systems), and monoclonal goat anti-human Tie-2 $(25 \mu \mathrm{g} / \mathrm{ml}, \mathrm{AF} 313, \mathrm{R} \& \mathrm{D}$ Systems).

Monoclonal mouse anti-CD34 (ready to use (RTU), clone QBEnd10, Dako A/S) and monoclonal mouse CD31 (ready to use (RTU), clone 1A10, NeoMarkers, Fremont, CA, USA) were used to identify endothelial cells. Monoclonal mouse anti-vimentin (RTU, clone V9, Dako A/S) was used to identify cells of mesenchymal origin, anti-cytokeratin 7 (1:100, clone OV-TL12/30, Dako A/S ) to identify trophoblast, anti- CD68 $(7.2 \mu \mathrm{g} / \mathrm{ml}$, clone PG-M1, Dako A/S) to identify Hofbauer cells. After 30 min incubation with blocking solution, sections were incubated with the primary antibodies at $4{ }^{\circ} \mathrm{C}$ overnight, followed by sequential $30 \mathrm{~min}$ incubations with biotinylated secondary antibodies (Dako A/S) and peroxidase labelled streptavidin. Immunohistochemistry was performed using a horseradish-peroxidase labelled streptavidin biotin (HRP-LSAB) kit (Dako A/S). The resulting signal was developed with diaminobenzidine (DAB) (Dako A/S). All incubations were performed in a moist chamber at room temperature, using PBS for washes between the incubation steps. The sections were counterstained with Mayer's hematoxylin solution (Merck, Darmstadt, Germany) and mounted with glycerol-gelatin (Sigma). Negative control stainings were performed by replacing the primary antibodies with the appropriate non-immune IgG or isotypes. To test the antigenic specificity of the antibodies, Tie- 1 and Tie- 2 primary antibodies were preincubated with their specific peptides $(10 \mu \mathrm{g} / \mathrm{ml}$ dilution for Tie-1P (C-18), and $10 \mu \mathrm{g} / \mathrm{ml}$ Tie-2P (C20), Santa Cruz Biotechnology) for $1 \mathrm{~h}$ at $37^{\circ} \mathrm{C}$. Then, the same protocol was applied. (Kayisli et al., 2006).

Photomicrographs were taken with an Axioplan microscope (Zeiss, Oberkochen, Germany). Tissue sections from different pregnancy days were evaluated for protein localization and intensity. All samples for each individual antibody were exposed to the same protocol and were stained using the same duration of staining.

The intensity of immunoreactivity was semi-quantitatively evaluated as follows. Positively stained cells were grouped according to the following categories: - (no staining) $1+$ (weak but detectable), $2+$ (moderate or distinct), $3+$ (intense). For each tissue, an HSCORE value was calculated by summing the percentages of cells grouped in one intensity category and multiplying this number with the weighted intensity of the staining, using the formula $\left[\mathrm{HSCORE}=\mathrm{P}_{\mathrm{i}}(\mathrm{i}+\mathrm{l})\right]$, where $\mathrm{i}$ represents the intensity scores and $\mathrm{P}_{\mathrm{i}}$ is the corresponding percentage of the cells. In each slide, five different areas were evaluated under a microscope using $400 \times$ original magnification, the percentage of the cells for each intensity within these areas was determined by two investigators who were blinded to slides and gestational age, and the average score was used (Demir et al., 2004).

Western blot technique. Western bloting procedures have been described in our recent studies (Demir et al., 2004; Kayisli et al., 2006) was applied. From placental tissues ( 3 samples for each gestational week; 8th week was addition for $\mathrm{WB}$ ), $\mathrm{n}=15$ ) total protein was extracted using a lysis buffer (50 mM HEPES, $\mathrm{pH} 7.4,150$ $\mathrm{mM} \mathrm{NaCl}, 10 \%$ glycerol, $1 \%$ Triton X-100, $1.5 \mathrm{mM} \mathrm{MgCl} 2,1 \mathrm{mM}$ EGTA, $100 \mathrm{mM} \mathrm{NaF}, 10 \mathrm{mM}$ sodium pyrophosphate and protease inhibitors, $1 \mathrm{mM} \mathrm{Na}_{3} \mathrm{VO}_{4}, 10 \mathrm{mg} / \mathrm{ml}$ leupeptin, $10 \mathrm{mg} / \mathrm{ml}$ aprotinin, and $4 \mathrm{mM}$ PMSF). The protein concentration was determined by a detergent compatible protein assay (Bio-Rad Laboratories,
Hercules, CA, USA). Samples $(20 \mu \mathrm{g})$ were loaded and separated by non-reducing SDS-polyacrylamide gel electrophoresis using $7.5 \%-12 \%$ Tris- $\mathrm{HCl}$ ready gels (Bio-Rad Laboratories) and electroblotted onto Hybond ECL nitrocellulose membrane (Amersham Pharmacia Biotech, Buckinghamshire, England). The membrane was blocked with $5 \%$ non-fat dry milk in TBS-T buffer $(0.05 \%$ Tween-20 in TBS, $\mathrm{pH}$ 7.4) for $1 \mathrm{~h}$ to reduce non-specific binding. Afterwards, the membrane was incubated for $1 \mathrm{~h}$ with monoclonal antibodies against VEGF (Santa Cruz Biotechnology), Flk-1 (Santa Cruz Biotechnology) and Flt-1 (Santa Cruz Biotechnology), and the polyclonal antibodies against Tie-1 (1/200; Santa Cruz Biotechnology) and Tie-2 (1/200; Santa Cruz Biotechnology). Following rinses with TBS-T for 15 min once and 10 min twice, the membranes were further incubated for $1 \mathrm{~h}$ with peroxidase labelled secondary antibodies (Vector Laboratories) diluted at 1:10000 and subsequently washed with TBS-T for 15 min once and $10 \mathrm{~min}$ twice. The immunoblot was developed using a chemiluminescent kit (NEN Life Science, Boston, MA). Equal loading was confirmed using Ponceau staining (Molecular Dynamics, Sunnyvale, $\mathrm{CA}$ ).

Statistical analysis. Statistical analysis for immunohistochemical and Western blot data were normally distributed (tested by Kolmogorov-Smirnov test) throughout the days of pregnancy tested. Analysis of variance (ANOVA) and the Tukey tests and pair wise multiple comparisons were carried out for statistical analysis. Statistical calculations were performed using the Statistical Package for social sciences (SPSS, Chicago, IL, USA) and Sigmastat for Windows, version 2.0 (Jandel Scientific Corporation, San Rafael, CA, USA).

\section{Results}

\section{Immunohistochemical observations}

Localization of VEGF and its receptors VEGFR-1 (Flt-1) and VEGFR-2 (Flk-1/KDR) proteins expression during the early pregnancy were examined by immunocytochemistry on serial sections analysis. Different anti-VEGF and anti- VEGFR-1 and VEGFR-2 polyclonal antibodies, and the polyclonal antibodies against Tie-1 and Tie-2 were used for each receptor.

\section{VEGF expression in the early placental tree}

VEGF immunoreactivity was mainly localized in cytotrophoblastic cell layer of placental villi and to angiogenic cell population forming primitive vascular structures and in some stromal cells in the villi core (Fig. 2a-d). The intensity of VEGF no immunostaining was seen when the primary antibody was replaced by preimmune rabbit IgG.

\section{VEGFR-1 (Flt-1) expression in the early pla- cental tree}

During the placental development, VEGFR-1 (Flt-1) immunoreactivity was detectable in the angiogenic cell cords (ACC) dominantly all of the primitive vascular tubes and angiogenic cells stained with CD31positive vascular structures (Fig. 1a and b). In the early 

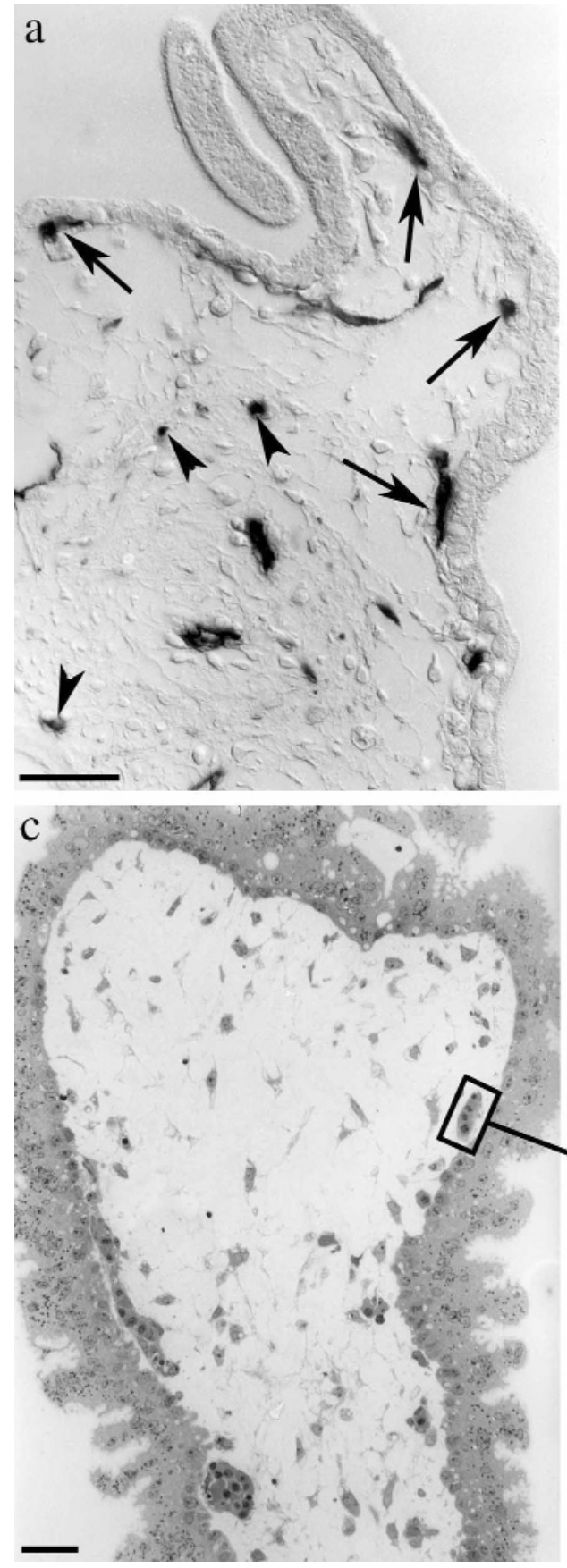
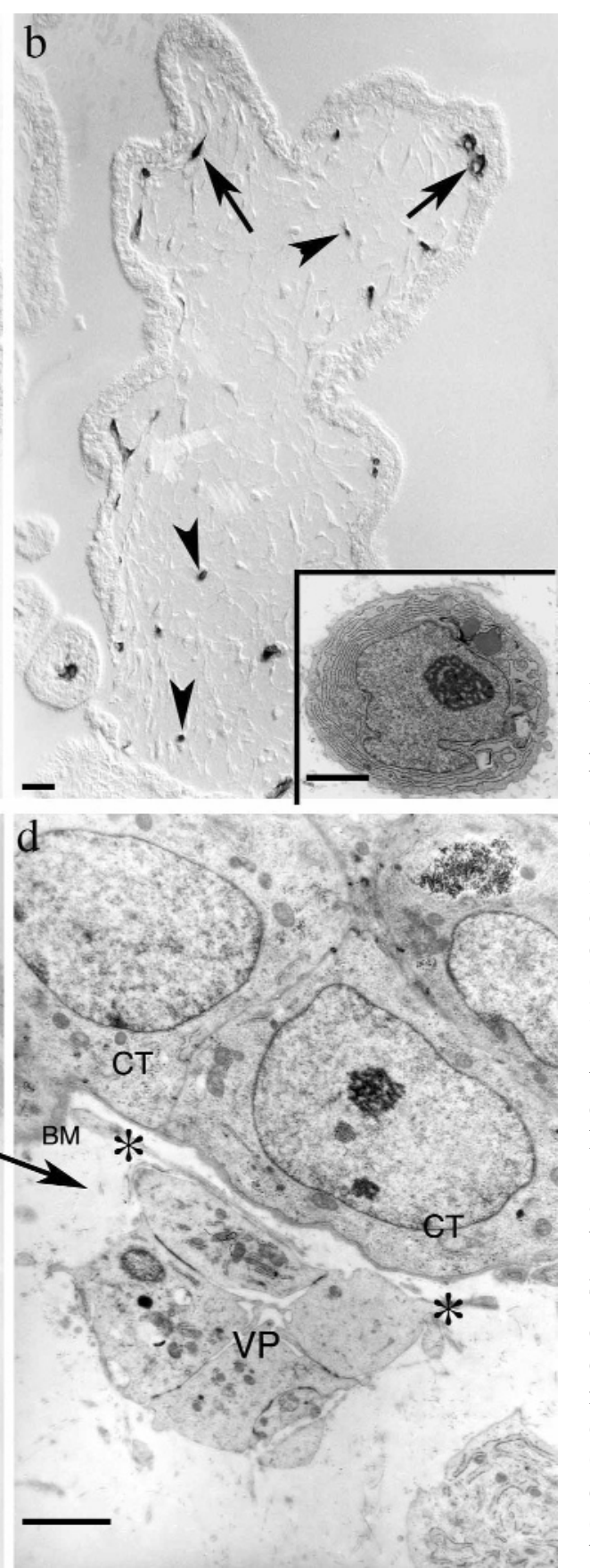

Fig. 1. Representative pictures showing first signs of vasculogenic patterns in mesenchymal villi. $(a, b)$ Single cells (arrowheads) or cell cords of vasculogenic patterns (arrows) visualized by CD31 immunoreactivity are often seen in close vicinity to the trophoblastic cover. (c, d) Cytoplasmic processes (asterisks in d) of the cells of vasculogenic patterns (VP) are seen in semithin sections extending towards the basement membrane (BM) of cytotrophoblast cells (CT) [(d) is the higher magnification of the area selected by the rectangle-connected arrow in (c)]. In the insert (b) the ultrastructure of a single haemangiogenic stem cell can be seen. These cells are characterized by centrically positioned nucleus with deep invaginations and a prominent nucleolus, and abundant rouge endoplasmic reticulum cisternae. (a) pregnancy day 37; (b) pregnancy day 27 ; (c, d) pregnancy day 22 p.c. (a, b, c) Scale bars $=50 \mu \mathrm{m}$; (insert $\mathrm{b}$ and d) scale bar $=4 \mu \mathrm{m}$. days of pregnancy (between days 22-28), most of the labeled ACC and primitive vascular tubes were not main vascular patterns (capillary network) that had not yet formed a lumen composed of elongated endothelial cells (Fig. 1a-d). Their presence was assessed by immunolabeling of serial tissue sections with several specific endothelial markers, the anti-CD31 (Fig. 1a, b). The intensity of the ACC and vascular patterns associated with immunostaining increased at the end of the villi maturation periods; including mesenchymal villi (MV) (Fig. 3a) that was differentiated into immature intermediate villi (IMIV) (Fig. 1a), and into to stem villi (SV) segments of the placental villi tree development when immunolabeling of VEGFR-1 was decreased observed both on large vascular tubes and on vascular patterns with a dilated lumen. The endothelium of a limited number of main vascular patterns localized in central areas of core in SV segment was lightly labeled throughout the placental development of early pregnancy.

During the early weeks (between 22-42 days) of pregnancy, there was an increase in the intensity of VEGFR-1-positive primitive vascular tube (connecting tubes) strands in SV and IMIV and fully constituted single angiogenic cells and ACC in MV and IMIV 


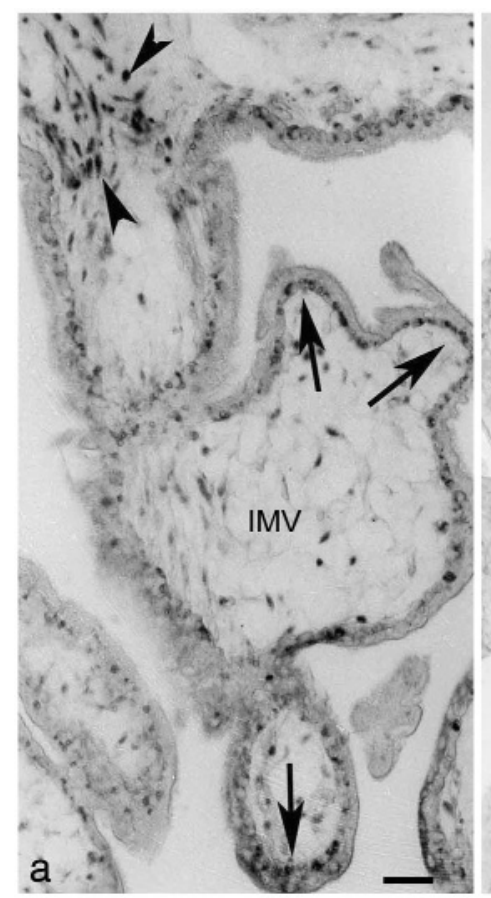

d

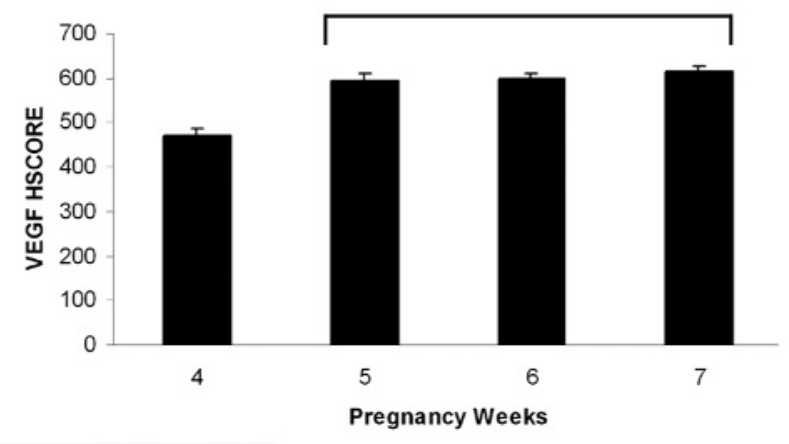

versus the staining (Fig. 3a). However, a similar increase in the intensity of CD31 the main vascular patterns structures were also observed. Consequently the degree of the intensity of VEGFR-1-positive vascular patterns to CD31 vascular structures was not significantly different between the IMIV and MV; with the exception of the matured segment of stem villi. The intensity of immunopositivity in SV was decreased when compared to IMIV and MV segments of placental villi tree. In the IMIV at the all days, a rich ACC and primitive vascular tube network comprising all of the vascular paterns strands and fully formed primitive connecting tubes expressed VEGFR-1, along with the endothelium of several main vascular structures localizing in central areas of IMIV. At the late days of pregnancy samples around day 42-48, there was a striking decrease in the immunolabeling intensity of the VEGFR-1-positive vascular patterns exhibited a dilated lumen on the period of pregnancy.
In addition to the findings in single angiogenic cell and ACC (Fig. 1a, b), we observed a strong VEGFR-1 immunolabeling on stromal cells especially in Hofbauer cells and also in cytotrophoblastic layer as moderate; and a lightly VEGFR-1 immunopositive in other connective tissue cells (Fig. 2a, b). This labeling decreased strongly due to segmental maturation of placental villi. The specific stromal cells population with a irregular shape, a excentric nucleus, and the vacuolated or un-vacuolated appearance and CD 68-positive phenotype of cells of the Hofbauer cells (placental macrophage) lineage were also immunoreactive for VEGFR-1 in the IMIV for al of the specimens examined (Fig. 2c).

Hofbauer cells were numerous in the IMIV (Fig. $2 \mathrm{c})$. Their distribution during the early days of pregnancy (between 22-48 days of pregnancy) were heterogeneous, and they were localized very near vascular patterns and close to ACC in small clusters in the 


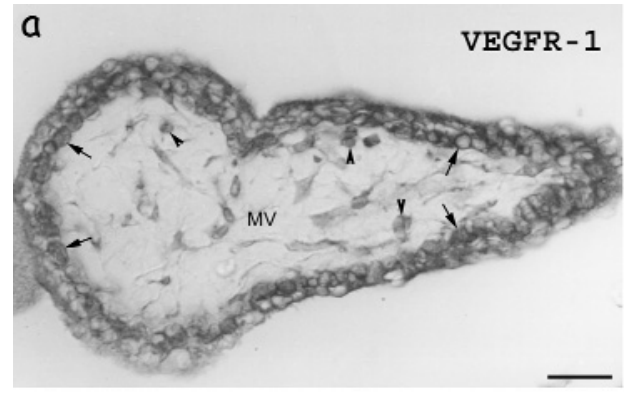

C

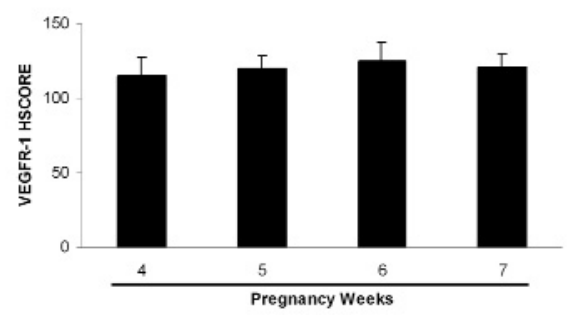

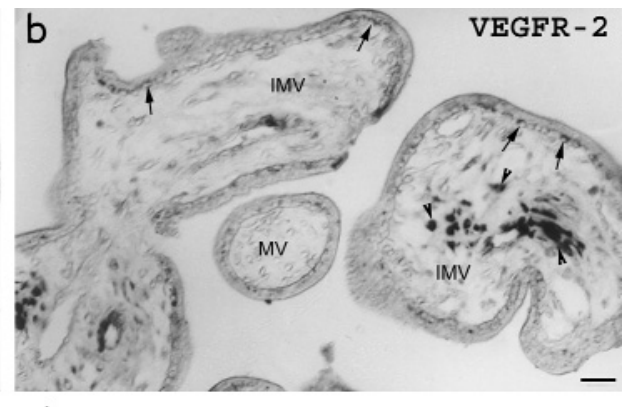

d

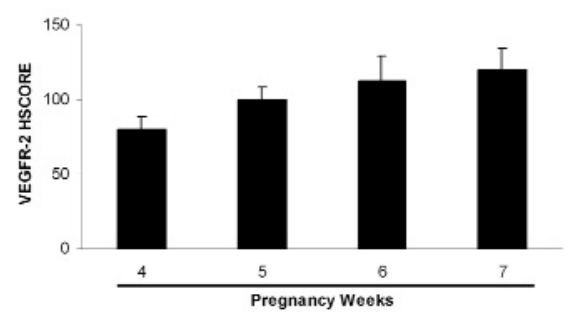

Fig. 3. Representative pictures showing expression of VEGFR-1 and VEGFR-2 in different cell types of the villous tree. (a) VEGFR-1 and (b) VEGFR-2 are expressed by cytotrophoblast (arrows) and by some stromal cells (arrow heads) in mesenchymal villi (MV) and immature intermediate villi (IMV). There were no significant changes in HSCORE evaluations of (c) VEGFR-1, and (d) VEGFR-2 throughout pregnancy weeks examined. (a) pregnancy day 27; (b) pregnancy day 37, p.c. Scale bar $=50 \mu \mathrm{m}$
IMIV; these cells, which secrete VEGF moderately (Fig. 2c), expressed VEGFR-1 receptors strongly (Fig. 3a). During the development of these segments of placental villi, other stromal cells and cytotrophoblastic layer were faintly immunolabeled, especially on the villi segments of MV and IMIV (Fig. 3a, b), particularly on days 22-42 of pregnancy.

\section{VEGFR-2 (Flk-1/KDR) expression in the vil- lous tree}

VEGFR-2 (Flk-1/KDR) was mainly localized to placental villi vascular structures. These are angiogenic cells and Hofbauer cells and non vascular components that were cytotrophoblastic layer and basic stromal cells. No immunolabeling was seen when the primary antibody was replaced by preimmune rabbit IgG.

VEGFR-2 vascular immunoreactivity was maximal during the placental villi vascularization processes, as shown by the higher intensity of stained vascular network in the villi segments involved in the maturation and complete vascularization, and the higher intensity degree of VEGFR-2-positive to CD31 positive vascular patterns in the placental villi tree.

During the vascular tube formation, VEGFR-2 expression was stronger than VEGFR-1 expression. At the beginning of the ACC formation in the MV, the majority of angiogenic cells strands and several primitive vascular tube sections were labeled with the antibody against VEGFR-2; the totality of the vascular structures was immunostained in the IMIV segments (Fig. $3 \mathrm{~b})$. These angiogenic cells and primitive vascular tube formation could be identified with anti-CD31 and CD34 antibodies (Fig. 1a, b). The intensity of VEGFR-2 staining persisted during the pregnancy advanced (Fig. 3d).

In the IMIV and MV, vascular patterns immunore- activity for VEGFR-2 was still observed; however, while the intensity of VEGFR-2 positive vessels with striking lumen was only slightly diminished, the intensity staining of vascular patterns expressing the receptor was considerably smaller owing to the overall increase in placental vascular density indicating with endothelial markers. The numbers of vascular patterns stained for VEGFR-2 and for VEGFR-1 were therefore similar during the same placental villi segments. The intensity of the immunolabeling was heterogeneous; it was lower in the SV segments, increased strongly in the IMIV and MV. At the beginning of the formation of MV, only a few angiogenic cells and some ACC faintly expressed VEGFR-2. These structures were more intensely immuno-labeled on ACC in IMIV segment of placental villi core (Fig. 3b).

Light labeling of stromal cells and of cytotrophoblastic layer were also observed at some specific segments of the placental villi. No significant labeling was found in the cells during the entire in the IMIV and MV (Fig. 3a, b); some intensely stained cells appeared, however, in the SV ongoing in maturation. Particularly striking was the immunolabeling of numerous stromal cells of villous tree, some of which had the appearance and immunophenotype for CD68, Hofbauer cells. The cytotrophoblastic layer was labeled with the anti-VEGFR-2 antibody, with an accentuation of the staining in the basal portion, particularly at days 24-28 of pregnancy (Fig. 3b).

\section{Tie-1 and Tie-2 expression in the villous tree}

Tie-1 and Tie-2 immunoreactivities were evaluated for villous types in placental villous tree including mesenchymal villi (MV) and immature intermediate villi (IMIV). These proteins were analyzed by Western blot 


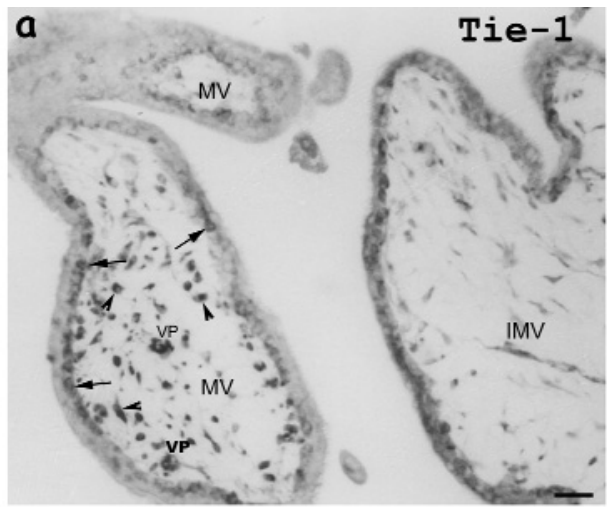

C

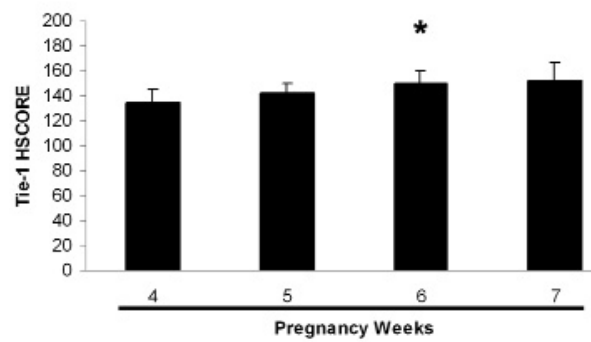

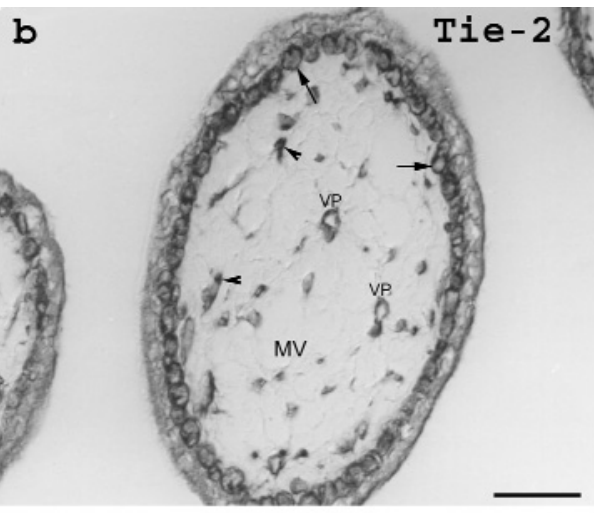

d

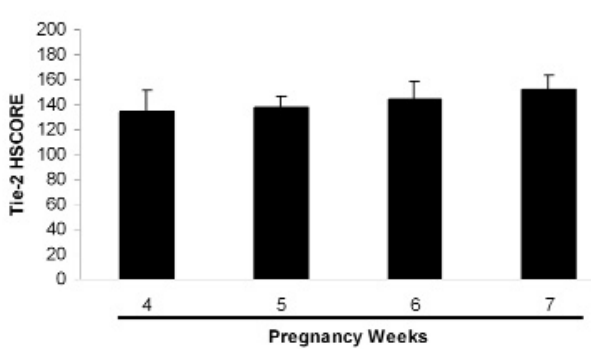

Fig. 4. Representative pictures showing expression of Tie-1 and Tie-2 in different cell types of the villous tree. (a) Tie-1 and (b) Tie-2 are expressed by cytotrophoblast (arrows) and by vasculogenic cell patterns (VP) in mesenchymal villi (MV) and immature intermediate villi (IMV). A strong immunoreactivity for Tie-2 was definitly seen in cytotrophoblastic cell layer. (c) Tie1 and (d) Tie-2 HSCORE evaluations revealed an increase as pregnancy progressed with a significant increase in Tie-1 on the $6^{\text {th }}$ week ( $<<0.05$ ). (a) pregnancy day 30; (b) pregnancy day 32 , p.c. Scale bar $=50 \mu \mathrm{m}$ technique. The intensity of immunoreactivities for Tie1 and Tie-2 was not change significantly in as pregnancy progressed. However, Tie-1 immunoreactivity was stronger than Tie- 2 in the stromal cells, but Tie-2 was strongest only in cytotrophoblastic layer in all pregnancy days evaluated (Fig. 4).

Tie-1 expresion: Cytotrophoblastic layer and various stromal cell varieties including fibroblast, angiogenic cells cords and Hofbauer cells, indifferent mesenchymal cells showed the Tie-1 immunoreactivity in MV and IMIV which was not alteration significantly as pregnancy progressed. Cytotrophoblastic cell layer and stromal cells in some villous segments was strong immunostainning for Tie-1, but the Hofbauer cells revealed a moderate immunoreactivity while indifferent mesenchymal cells displayed weak staining and no significant alteration was observed as pregnancy progressed. Due to the segmental villous maturation Tie-1 expresion was decreased in vascular endothelium when compared with the angiogenic cell cords in all pregnancy weeks evaluated (Fig. 4a, c). The intensity of Tie-1 immunoreactivity was gradually decreased from MV towards SV in parallel to villous maturation but not to the weeks of pregnancy (Fig. 4a, c).

Tie-2 expresion: The main expresion sourse for Tie-2 was definitly cytotrophoblastic cell layer in MV. A strong immunoreactivity was observed in these cells lined viili trofoblast and villi stroma including angiogenic cell cords exhibited a moderate immunoreactivity for Tie-2 without significant alterations related to the week of pregnancy. Due to the villi maturation and development of placental villi large vascular paterns were situated in central area of the aged villi. The vascular endothelium showed a decreasing from MV to IMIV type for Tie-2. The cellular diversity with different types in stroma displayed a gradual increasing from absent to moderate for Tie-2 immunoreactivity as pregnancy advanced (Fig. 4b, d).

Western blotting analysis of tie- 1 and tie- 2 were showed, in general, a similar reaction in all of the gestational days examined. Additional samples for western blotting between 50-56 days were analyzed. There was an increase reaction for tie-1 between gestational days of 50-56 examined; however this increasing was not in significant difference for all of gestational days. (Fig. 5)

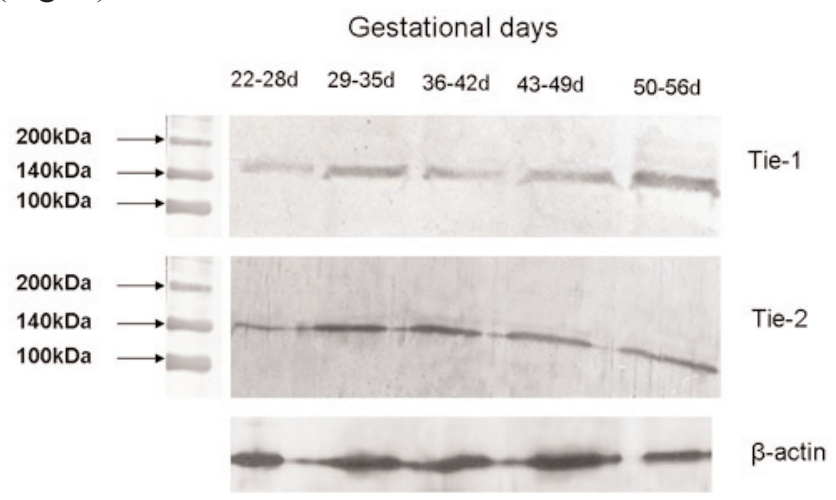

Fig. 5. This Fig. is showing the western blotting analysis of tie-1 and -2 expression in early days of pregnancy. The immunoblot signals at around $120 \mathrm{kDa}$ (for Tie-1) and $140 \mathrm{kDa}$ (for Tie-2) were detected. They showed a little gradual increase in expresssion as pregnancy progressed. No statistically significant differences can be observed from the tie-1 and-2 bands when compared with $\beta$-actin bands. 


\section{Discussion}

Our previous study on the expression of placental VEGF and its receptors, VEGFR-1 (Flt-1) and VEGFR-2 (Flk-1/KDR) has been carried out in very early pregnancies with various gestational days (Demir et al., 2004). In the present study we aimed to determine, the expression profile of VEGF and its receptors, VEGFR-1 and-2 with angiopoietin receptor Tie-1 and Tie-2; and to evaluate the molecular and morphological steps during vasculogenesis and angiogenesis in the early human placenta.

The expression of the VEGF receptors that mediate the functionality not only of the VEGF-A, but also of other components of the VEGF family (PIGF, VEGFB, VEGF-C, VEGF-D). To perform a complete study on the expression of the VEGF and its receptors, with angiopoietin receptor Tie-1 and Tie-2; we used different investigation methods, such as immunohistochemistry, electron microscope and Western blot.

The findings obtained in the very early placental samples were compared with those observed in tissue samples of late early days of pregnancies.

The immunohistochemistry results showed that VEGF and its receptors VEGFR-1 and VEGFR-2 were not localized in all of the placental components was detected only in the cytotrophoblast cells lined the villi surface, in the ACC cords, in Hofbauer cells and preendothelial cells forming primitive vascular patterns. These findings are partially in agreement with previous investigations (Cooper et al., 1995; Clark et al., 1998; Cheung, 1997; Shore et al., 1997; Dunk and Ahmed, 2001).

The location of VEGFR-1 and VEGFR-2 in the placenta early days (between 22-35 p.c.) of pregnancy, was the same with respect to the late days (between $32-48$ p.c.) of pregnancy. In the maturation period advanced in some villi, particularly in stem villi type, some segments of trophoblast and/or some vessels and/or clusters of stromal cells did not express the VEGF and its receptors, as demonstrated by the immunohistochemical observation in each MV and IMIV. The intensity of immunoreactivity of some positive components was significantly lower in all the pregnancy days, particularly in the late days of the examined. As regards the VEGFR-2, its expression was detected, not only in the primitive vascular structures, but also in the cytotrophoblastic layer and in the stromal cells. However, the ACC in MV and IMIV of all the days of pregnancy showed a significant higher intensity of immunoreactivity with respect to the matured stem villi segments.

The Western blotting technique shoved that, the protein levels of VEGF and its receptors VEGFR-1, VEGFR-2 in the all examined gestational age, no difference showed a statistically significant when compared with the each other.
As regards the proteins expression levels, the data showed a decrease of all the Tie- 1 and Tie- 2 receptors from the 4 weeks placentas to the 7 weeks even if not always significantly. The findings of this study demonstrated that the expression of the VEGF and its receptors and angiopoietin receptors Tie- 1 and Tie- 2 seem to be related to the different degree of pregnancy days.

According to our results, the angiopoietin receptors Tie-1 and -2 showed a higher expression levels in respect to other study factors. The receptors protein levels increased from the early days to the advanced days of gestation, but this alteration was not significant. The intensity of the immunolabeling for these proteins were not significant compared to each other of gestation days were examined.

Our knowledge regarding the placental expression of the VEGF and its receptors in the pathological disorders is discordant. In fact that studies carried out on placentas from pathological pregnancies complicated by different degrees of clinical severity, showed that the hypoxic environment up-regulates VEGF and its receptors (Helske et al., 2001; Kumazaki et al., 2002; Tsatsaris et al., 2003). On the contrary, some authors demonstrated that VEGF and VEGFR-1 are down-regulated in placentas of pregnancies with severe pathological conditions (Zhou et al., 2002), and studies about the influence of hypoxia on placental tissue have demonstrated that VEGF-A is up-regulated, while VEGFR-1 is down-regulated (Ahmed et al., 2000). Moreover, some investigations have showed that an increase in VEGF-A, together with other factors, may lead to the structural alterations involved in the functional processes of the placental tissue (Brockelsby et al., 2000; El-Salahy et al., 2001; Redman and Sargent, 2001). From these discordant data it could be hypothesized that an overproduction of VEGF-A in a definite period of gestation might lead to the alteration of the placental tissue, and that only the placental undamaged components might continue to overproduce VEGF-A (Sgambati et al., 2004).

In the placenta of pregnancies from very early days, a compensatory mechanism against the hypoxic environment should be occurs, with an increasing production of all the receptors levels and of the placental components that produce VEGF and its receptors. It was suggested that the mRNA levels of the receptors are not paralleled by the protein expression, as revealed by immunohistochemistry and by western blotting (Sgambati et al. 2004). According to alteration of protein synthesis for VEGF and its receptors, it is possible to be the slight functional changes of the placental components. In the cases of full term human placental barrier, a more severe structural complication characterized by evident ultrastructural alterations (Demir 1979, 1980), only some placental components might be able to make a function like-nefro-pneumoid units for transportation 
and secretion of some substances (Demir et al.1995).

Due to the different pathological condition, the immunohistochemistry showed that in the some components of placenta were positive for VEGF and its receptors (Tsokos et al., 2002; Zhou et al., 2002). It was possible to hypotheses that there is probably damage in the placental components involved in as triggers by a hypoxic environment. Due to this structural damage during placental development, as a similarity, it is possible to elicit an over expression for VEGF and its VEGFR-1 and VEGFR-2 by the placental elements, so, it is possible that partially damaged, in the attempt of a compensatory mechanism for placental health vascularization.

The first vasculogenesis in the chorionic villi tree starts at days 21 post conception, during the stage of a 4 somite embryo. At this stage, in the villi core, haemangiogenic stem cells derived from mesenchymal cells differentiate prior to the formation of first vessels rather than originating from blood monocytes (Demir et al., 1989). In the attempt to clarify the disagreements of the data on the placental VEGF and its receptors affected angiogenesis and vasculogenesis.

We have shown that a variety of factors are associated with placental angiogenesis and vasculogenesis. Under the light of our and other data this study focuses on describing an integrated view on VEGF and its some receptors in early placental of pregnancy.

The results of this study are partially in agreement with previous studies on the expression of VEGF and its receptors in placenta of pregnant with normal pregnancy (Ahmed et al. 2000) and not accordance pregnant with pathological disorders (Helske et al., 2001; Kumazaki et al., 2002; Tsatsaris et al., 2003 Sgambati et al., 2004). As reported in our recently study on the VEGF-A and its receptors (Demir et al., 2004) and anti angiopoietin receptors Tie-1 and Tie-2 expression (Kayişli et al., 2006), the disagreements might be due to an assemblage in the same study group cases of pathological disorders with different degree of clinical severity.

In conclusion, we demonstrated that in the placental tissue from very early pregnancies there is a dysregulation of the expression of the VEGF and its family receptors 1 and 2 and angiopoietin receptors Tie-1 and Tie-2 related to the different degree of villi type in developmental severity stages. However, the VEGF and its receptors with angiopoietin receptors Tie-1 and Tie-2 seem to play an important role in the placental vascularization during the pregnancy. We believe that, it should be necessary to perform studies also on the expression of other factors of the VEGF family, as well as of the receptor VEGFR-1s (soluble) that has been shown to be up regulated in pathological conditions (Helske et al., 2001; Kumazaki et al., 2002; Tsatsaris et al., 2003).
The functional investigations may be establish a communication between placental villi angiogenesis and vasculogenesis during early days of pregnancy, and probably to shove a relationship with the role played by all the members of VEGF family in the gestational complications of different clinical severity.

Acknowledgements: eThis study was partly funded by a grant from Akdeniz University Research Foundation and Technical Research Council of Turkey (TUBITAK) (project no: SBAG3267-105S460)

\section{References}

Ahmed A, Dunk C, Ahmad S and Khaliq A. Regulation of placental vascular endothelial growth factor (VEGF) and placenta growth factor (PIGF) and soluble Flt-1 by oxygen - a review. Placenta 2000; 21: 16-24.

Benirschke K, Kaufmann P. Pathology of the Human Placenta. Springer, New York, 2000.

Brockelsby JC, Anthony FW, Johnson IR and Baker PN. The effects of vascular endothelial growth factor on endothelial cells: A potential role in preeclampsia. Am. J. Obstet. Gynecol. 2000;182: 176-83.

Cervar M, Blaschitz A, Dohr G and Desoye G. Paracrine regulation of distinct trophoblast functions in vitro by placental macrophages. Cell Tissue Res. 1999; 295: 297-305.

Charnock-Jones DS, Kaufmann P and Mayhew TM. Aspects of human fetoplacental vasculogenesis and angiogenesis. I. Molecular regulation. Placenta 2004; 25: 103-13.

Cheung CY. Vascular endothelial growth factor: possible role in fetal development and placental function. J Soc Gynecol Invest. 1997; 4.169-77.

Clark DE, Smith SK, Licence D, Evans AL and Charnock-Jones DS. Comparison of expression patterns for placenta growth factor, vascular endothelial growth factor (VEGF), VEGF-B and VEGF-C in the human placenta throughout gestation. J Endocrinol. 1998; 159: 459-67.

Cogle CR and Scott EW. The hemangioblast: cradle to clinic. Exp Hematol. 2004; 32: 885-890.

Cooper JC, Sharkey AM, McLaren J, Charnock-Jones DS and Smith SK. Localization of vascular endothelial growth factor and its receptor, flt, in human placenta and decidua by immunohistochemistry. J Reprod Fertil . 1995;105: 205-13.

Demir R. Ultrastructure of the epithelium of the chorionic villi of the human placenta. Acta Anat (Basel). 1980; 106: 18-29.

Demir R, Demir N, Ustunel I, Erbengi T, Trak I and Kaufmann P. The fine structure of normal and ectopic (tubal) human placental villi as revealed by scanning and transmission electron microscopy. Zentralbl Pathol. 1995; 140: 427-42.

Demir R and Erbengi T. Some new findings about Hofbauer cells in the chorionic villi of the human placenta. Acta Anat (Basel).1984;119: 18-26

Demir R, Kaufmann P, Castellucci M, Erbengi T and Kotowski A. Fetal vasculogenesis and angiogenesis in human placental villi. Acta Anat (Basel) 1989; 136: 190-203.

Demir R, Kayisli UA, Cayli S and Huppertz B. Sequential Steps During Vasculogenesis and Angiogenesis in the Very Early Human Placenta. Placenta 2006; 27:535-39.

Demir R, Kayisli UA, Celik-Ozenci C, Korgun ET, DemirWeusten AY and Arici A. Structural differentiation of human uterine luminal and glandular epithelium during early pregnancy: an ultrastructural and immunohistochemical study. Placenta 2002; 23: 672-84.

Demir R, Kayisli UA, Seval Y, Celik-Ozenci C, Korgun ET, Demir-Weusten AY and Huppertz B. Sequential expression of 
VEGF and its receptors in human placental villi during very early pregnancy: differences between placental vasculogenesis and angiogenesis. Placenta 2004; 25:560-72.

Demir R, Kosanke G, Kohnen G, Kertschanska S and Kaufmann P. Classification of human placental stem villi: review of structural and functional aspects. Microsc Res Tech. 1997; 38: 2941.

Demir R, Acar G, Tanriover G, Seval Y, Kayisli UA, Agar A. Effects of excess vitamin B6 intake on cerebral cortex neurons in rat: an ultrastructural study. Folia Histochem Cytobiol. 2005; 43(3):143-50.

Demir R, Seval Y, Huppertz B. Vasculogenesis and angiogenesis in the early human placenta. Acta Histochem. 2007;109 (4):25765 .

Dunk C and Ahmed A. Expression of VEGF-C and activation of its receptors VEGFR-2 and VEGFR-3 in trophoblast. Histol Histopathol. 2001; 16: 359-75.

Dvorak HF, Brown LF, Detmar M and Dvorak AM. Vascular permeability factor/vascular endothelial growth factor, microvascular hyperpermeability, and angiogenesis. Am J Pathol. 1995; 146: 1029-39.

El-Salahy EM, Ahmed MI, El-Gharieb A and Tawfik H. New scope in angiogenesis: Role of vascular endothelial growth factor (VEGF), NO, lipid peroxidation, and vitamin $\mathrm{E}$ in the pathophysiology of pre-eclampsia among Egyptian females. Clin Biochem. 2001; 34: 323-29.

Ferrara N. Vascular endothelial growth factor and the regulation of angiogenesis. Recent Prog Horm Res 2000; 55: 15-35.

Ferrara N. Vascular endothelial growth factor: basic science and clinical progress. Endocr Rev. 2004; 25 581-611.

Fong GH, Rossant J, Gertsenstein M and Breitman ML. Role of the Flt-1 receptor tyrosine kinase in regulating the assembly of vascular endothelium. Nature 1995; 376: 66-70.

Fong GH, Zhang L, Bryce DM and Peng J. Increased hemangioblast commitment, not vascular disorganization, is the primary defect in flt-1 knock-out mice. Development 1999;126: 3015-25.

Hanahan D. Signaling vascular morphogenesis and maintenance. Science 1997;277: 48-50.

Hanahan D and Folkman J. Patterns and emerging mechanisms of the angiogenic switch during tumorigenesis. Cell 1996; 86: 353-64.

Helske S, Vourela P, Carpén O, Horning C, Weich H and Halmesmäki E. Expression of vascular endothelial growth factor receptors 1, 2 and 3 in placentas from normal and complicated pregnancies. Mol. Hum. Reprod. 2001; 7: 205-10.

Hiratsuka S, Nakao K, Nakamura K, Katsuki M, Maru Y and Shibuya M. Membrane fixation of vascular endothelial growth factor receptor 1 ligand-binding domain is important for vasculogenesis and angiogenesis in mice. Mol Cell Biol. 2005; 25: 346-54.

Gu B, Alexander JS, Gu Y, Zhang Y, Lewis DF and Wang Y. Expression of lymphatic vascular endothelial hyaluronan receptor-1 (LYVE-1) in the human placenta. Lymphat Res Biol. 2006; 4: 11-7.

Joukov V, Pajusola K, Kaipainen A, et al. A novel vascular endothelial growth factor, VEGF-C, is the ligand for the Flt-4 receptor tyrosine kinase (VEGFR-3) EMBO J. 1996; 15: 29098.

Kaufmann P, Mayhew TM and Charnock-Jones DS. Aspects of human fetoplacental vasculogenesis and angiogenesis. II. Changes during normal pregnancy. Placenta 2004; 25: 11426.

Kayisli UA, Cayli S, Seval Y, Tertemiz F, Huppertz B and Demir R. Spatial and temporal distribution of Tie-1 and Tie-2 during very early development of the human placenta. Placenta 2006; 27:648-59.

Kingdom J, Huppertz B, Seaward G and Kaufmann P. Develop- ment of the placental villous tree and its consequences for fetal growth. Eur J Obstet Gynecol Reprod Biol. 2000; 92: $35-43$.

Kumazaki K, Nakayama M, Suehara N and Yoshinao W. Expression of vascular endothelial growth factor, placental growth factor, and their receptors flt- 1 and KDR in human placenta under pathologic conditions. Hum. Pathol. 2002; 33: 1069-77.

Leach L, Babawale MO, Anderson M and Lammiman M. Vasculogenesis, angiogenesis and the molecular organisation of endothelial junctions in the early human placenta. J Vasc Res. 2002; 39: 246-59.

Maisonpierre PC, Suri C, Jones PF, et al. Angiopoietin-2, a natural antagonist for Tie 2 that disrupts in vivo angiogenesis. Science 1997; 277: 55-60.

Mayhew TM, Charnock-Jones DS and Kaufmann P. Aspects of human fetoplacental vasculogenesis and angiogenesis. III. Changes in complicated pregnancies. Placenta 2004; 25: 12739.

Millauer B, Wizigmann-Voos S, Schnurch H, et al. High affinity VEGF binding and developmental expression suggest Flk-1 as a major regulator of vasculogenesis and angiogenesis. Cell 1993;72: 835-46.

O'Rahilly R and Tucker JA. The early development of the larynx in staged human embryos. I. Embryos of the first five weeks (to stage 15). Ann Otol Rhinol Laryngol. 1973; 82:1-27.

Pardanaud L, Yassine F and Dieterlen-Lievre F. Relationship between vasculogenesis, angiogenesis and haemopoiesis during avian ontogeny. Development 1989;105: 473-85.

Park JE, Chen HH, Winer J, Houck KA and Ferrara N. Placenta growth factor. Potentiation of vascular endothelial growth factor bioactivity, in vitro and in vivo, and high affinity binding to Flt-1 but not to Flk-1/KDR. J Biol Chem. 1994; 269: 25646-54.

Redman CW and Sargent IL. The pathogenesis of pre-eclampsia. Gynecol. Obstet. Fertil. 29, 518-522.

Sato TN, Tozawa Y, Deutsch U, et al. Distinct roles of the receptor tyrosine kinases Tie-1 and Tie-2 in blood vessel formation. Nature 1995; 376:70-74.

Sgambati E, Marini M, Zappoli Thyrion GD, et al. VEGF expression in the placenta from pregnancies complicated by hypertensive disorders. BJOG Int J Obstet Gynecol. 2004; 111: 564-70.

Seval Y, Korgun ET, Demir R. Hofbauer cells in early human placenta: possible implications in vasculogenesis and angiogenesis. Placenta. 2007;28 (8-9):841-5.

Senger DR, Galli SJ, Dvorak AM, Perruzzi CA, Harvey VS and Dvorak HF. Tumor cells secrete a vascular permeability factor that promotes accumulation of ascites fluid. Science 1983;219: 983-85.

Shalaby F, Rossant J, Yamaguchi TP, et al. Failure of blood-island formation and vasculogenesis in Flk-1-deficient mice. Nature 1995;376: 62-6.

Shore VH, Wang TH, Wang CL, Torry RJ, Caudle MR and Torry DS. Vascular endothelial growth factor, placenta growth factor and their receptors in isolated human trophoblast. Placenta $1997 ; 18: 657-65$.

Suri C, Jones PF, Patan S, et al. Requisite role of angiopoietin-1, a ligand for the TIE2 receptor, during embryonic angiogenesis. Cell 1996; 87: 1171-80.

Szukiewicz D, Szewczyk G, Watroba M, Kurowska E and Maslinski S. Isolated placental vessel response to vascular endothelial growth factor and placenta growth factor in normal and growth-restricted pregnancy. Gynecol Obstet Invest. 2005; 59: 102-7.

Tammela T, Enholm B, Alitalo K and Paavonen K. The biology of vascular endothelial growth factors. Cardiovasc Res. 2005; 65: 550-63.

Terman BI, Dougher-Vermazen M, Carrion ME, et al. Identifica- 
tion of the KDR tyrosine kinase as a receptor for vascular endothelial cell growth factor. Biochem Biophys Res Commun. 1992; 187: 1579-86.

Torry DS and Torry RJ. Angiogenesis and the expression of vascular endothelial growth factor in endometrium and placenta. Am J Reprod Immunol. 1997;37:21-9.

Tsatsaris V, Goffin F, Munaut C, et al. Overexpression of the soluble vascular endothelial growth factor receptor in preeclamptic patients: pathophysiological consequences. J Clin Endocrinol Metab. 2003; 88: 5555-63.

Tsokos M, Longauer F, Kardosova V, Gavel A, Anders S and Schulz F. Maternal death in pregnancy from HELLP syndrome. A report of three medico-legal autopsy cases with special reference to distinctive histopathological alterations. Int $\mathrm{J}$ Legal Med. 2002;116: 50-3.
Vuckovic M, Ponting J, Terman BI, Niketic V, Seif MW and Kumar S. Expression of the vascular endothelial growth factor receptor, KDR, in human placenta. J Anat. 1996; $188(\mathrm{Pt}$ 2): 361-66.

Vuorela P, Hatva E, Lymboussaki A, et al. Expression of vascular endothelial growth factor and placenta growth factor in human placenta. Biol Reprod. 1997; 56: 489-94.

Zhou Y, McMaster M, Woo K, et al. Vascular endothelial growth factor ligands and receptors that regulate human cytotrophoblast survival are dysregulated in severe preeclampsia and hemolysis, elevated liver enzymes, and low platelets syndrome. Am. J. Pathol. 2002; 160: 1405-23. 